\title{
Vortices Street Analysis of a Prionace Glauca Pectoral Fin in Unsteady Flow
}

\author{
Qingsong $\mathrm{Hu}^{*}$, Xiwu Liu and Wenping Cheng \\ College of Engineering, Shanghai Ocean University, Shanghai, 201306, China \\ *Corresponding author
}

\begin{abstract}
Prionace glauca has high efficiency and long-distance cruising mode, its pectoral fins play an important role in keeping balance and stability. Selecting the pectoral fins as a breakthrough to conduct related research has an important value for the optimization design of autonomous underwater vehicle (AUV) and other marine equipment. Through numerical description, the establishment of a two-dimensional fine pectoral model and finite element calculations in the unsteady fluid, we found the variation features of Karman Vortex Street of flexible pectoral fins in the unsteady flow. Further comparative analysis of vortex characteristics of Prionace glauca pectoral fins models with different shapes and under the same boundary conditions, the study found that the streamlined samples of pectoral fins help reduce the impact of water flow and improve the stability of Prionace glauca during swimming in the flow field, which provides a good foundation for structural optimization design and other related applications of AUV.
\end{abstract}

Keywords-Prionace glauca; pectoral fins; finite element; Karman Vortex Street

\section{INTRODUCTION}

There exist currents and other forms of energy in the ocean. Prionace glauca which is a typical representative of sharks and has the characteristics of swimming posture stability, high efficiency, long distance cruising, has survived for millions of years on earth. Currently, Autonomous underwater vehicle (AUV) has an extensive application in underwater exploration and other fields, and is the forefront of Marine power competition [1,2]. Prionace glauca pectoral has a very important function in keeping equilibrium and stability during swimming, and there is a corresponding structure in AUV. Pectoral fins elasticity surface characteristics, proper shrinkage and bending in the water is very crucial to efficiently complete the work [3]. AUV still can't rely on simulation to reach a more efficient level, which attributes to the deficient study of pectoral fins mechanics mechanism in the impact process of water.

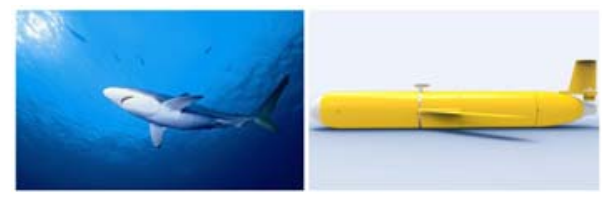

FIGURE I. THE STRUCTURE CONTRAST OF PRIONACE GLAUCA AND AUV
Since the 90s of the last century, the research of dynamics promoting mechanism based on fluid-structure interaction has become the focus[4,5], and more researches have studied fish propulsive wave parameters and the effect of fins shape on propulsion performance by utilizing the "three-dimensional fluctuation board" and other methods and simplifying the structure of the fish [6,7]. In recent years, the studies has focused on the drag reduction mechanism of surface trench and shield rib structure of shark [8], but the stability of the prionace glauca swimming is still lacking of in-depth study results. According to the above research status, this paper select prionace glauca as the research object. We numerically described the physical features of prionace glauca and established sophisticated cross section model of two-dimensional pectoral. Then we operated the infinite calculation in the unsteady flow and analyzed the variation features of Karman Vortex Street of flexible pectoral fins in the unsteady flow, which can provide support for the optimal design of AUV and other Marine equipment.

\section{Two-Dimensional Modeling Of Prionace GLAUCA PECTORAL}

Pectoral fins play the most important role in keeping balance of Prionace glauca during swimming. This study mainly relates to two-way coupling vortex characteristics of Prionace glauca pectoral fins in the flow field, and therefore it is needed to focus on describing shape characteristics of Prionace glauca pectoral fins. According to the measurement of a Prionace glauca specimen, pectoral fins' characteristics are shown in Table 1, we can find that the total length of pectoral fins is $440.6 \mathrm{~mm}$, pectoral fins' maximum width is $190.5 \mathrm{~mm}$, its horizontal abduction angle is 5 degrees, and the top of pectoral fins shrink slightly backward. Since this study only considers calculation of two-dimensional model, thus the impact of attack angle is ignored. This study examines vortex characteristics of Prionace glauca pectoral fins during exercise, which is based on the fluid-structure coupling physics research technology, so detailed description of pectoral fins' shape is very crucial. According to the outline dimensions of Prionace glauca pectoral fins, two-dimensional coupling calculation model of pectoral fins in the flow field can be built, as shown in Figure 2. 
TABLE I. ONE PRIONACE GLAUCA PECTORAL MAIN FEATURES DESCRIPTION

\begin{tabular}{|c|c|}
\hline Feature Name & values \\
\hline The pectoral fin length & $440.6[\mathrm{~mm}]$ \\
\hline the maximum width of pectoral fin & $190.5[\mathrm{~mm}]$ \\
\hline the horizontal abduction angle & 5 degrees \\
\hline
\end{tabular}

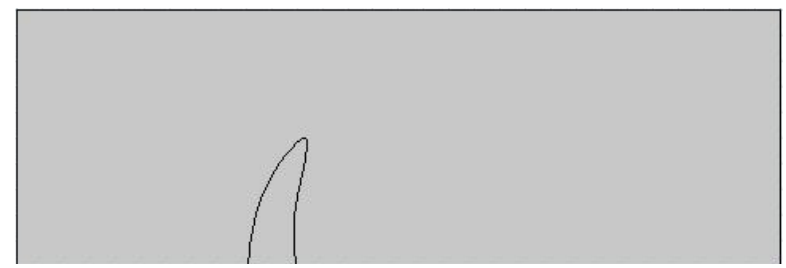

FIGURE II. TWO-DIMENSIONAL COUPLING CALCULATION MODEL OF PECTORAL FINS

\section{NUMERICAL CALCULATION OF PRIONACE GLAUCA PECTORAL}

\section{A. The Meshing of Computing Domain}

In this study, we utilized the finite element method to carry out fluid-structure interaction simulation analysis of Prionace glauca pectoral fins, thus the rational crucial meshing is of vital importance. This study divided a rectangular computational domain into tetrahedral mesh, and encrypted the connecting parts of pectoral fins' top and boundary. The above work enhance the accuracy of the simulation results, Figure 3 shows the results of computational domain meshing.

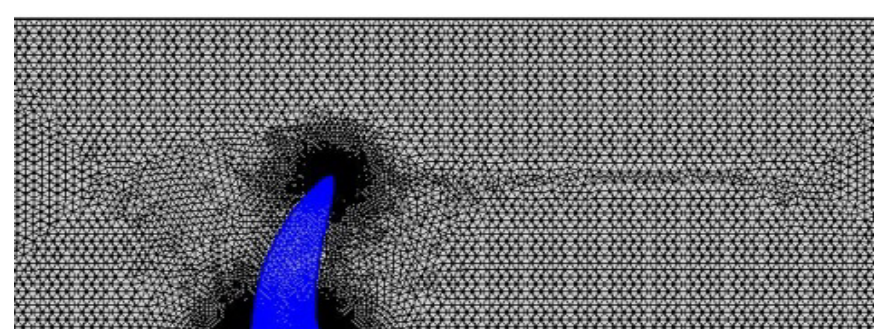

FIGURE III. THE RESULTS OF COMPUTATIONAL DOMAIN MESHING

\section{B. Numerical Simulation Calculation of Prionace Glauca Pectoral Fins}

Prionace glauca lives in the ocean, so we chose the incompressible fluid model to analyze mutual coupling of pectoral fins and water flow. Firstly, variation characteristics of Karman Vortex street of pectoral fins made by rubber material in the unsteady fluid were analyzed. The flow direction is perpendicular to the entrance, and the inlet velocity is $V$, duration is $6 \mathrm{~s}$.

$$
V=\frac{U t^{2}}{\sqrt{t^{4}-0.06 t^{2}+0.0016}}
$$

where $U$ is the average inlet velocity, this calculation $U=0.1 \mathrm{~m} / \mathrm{s}, t$ is the time, the image of the entry speed is shown in Figure 4.

Physical parameters of the rubber material are shown in Table 2, the elastic moduleus is $7.84 \mathrm{MPa}$, the shear modulus is $2.9 \mathrm{GPa}$, the density is $1000 \mathrm{~kg} / \mathrm{m}^{3}$, Poisson's ratio is 0.47 . The contraction deformation adaptability of Prionace glauca pectoral fins under the impact of unsteady currents can effectively reduce the impact of ocean currents, and enhance the swimming stability. This study selected six vortex diagrams of six time points ,as shown in Figure 6, respectively, for the time $\mathrm{t}=1 \mathrm{~s}, \mathrm{t}=2 \mathrm{~s}, \mathrm{t}=3 \mathrm{~s}, \mathrm{t}=4 \mathrm{~s}, \mathrm{t}=5 \mathrm{~s}, \mathrm{t}=6 \mathrm{~s}$; through analyzing vortex diagrams, we can see that ovortex flow is growing with the increase of the flow velocity.

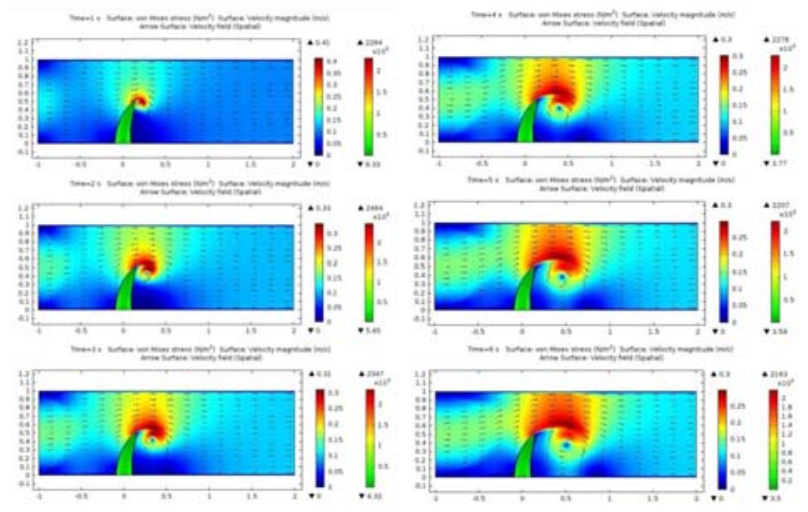

FIGURE IV. COMPARISON OF SIX TIME POINTS’ VORTEX

TABLE II.PHYSICAL PARAMETERS OF THE RUBBER MATERIAL

\begin{tabular}{|c|c|}
\hline Parameters & Value \\
\hline elasticity modulus & $7.84[\mathrm{Mpa}]$ \\
\hline Poisson's ratio & 0.47 \\
\hline Density & $1000\left[\mathrm{Kg} / \mathrm{m}^{\wedge} 3\right]$ \\
\hline Shear elasticity & $2.9[\mathrm{Gpa}]$ \\
\hline
\end{tabular}

\section{The Effect of Pectoral Fins' Shape Features on the Stability of Prionace Glauca}

To analyze the effect of shape characteristics of pectoral fins on the stability of Prionace glauca in the fluid, the study changed streamlined characteristics of pectoral fins, and compared it with pectoral fins specimen of model A, as shown in Figure 5. We set boundary conditions of the fluid simulation in the Model B same as Model A, and compared the vortex diagrams and pressure diagrams of the two models at the same time node, as shown in Figure 6, study shows that the stress of roots of pectoral fins of model B is larger, and the amount of displacement of pectoral fins' top in the $\mathrm{X}$-axis direction is more larger. The study compared the resistance change of two models suffered in computational fluid dynamics, as shown in Figure 7, the impact that specimen pectoral fins suffered in the fluid is significantly less than model B, with little fluctuation and strong stability. 

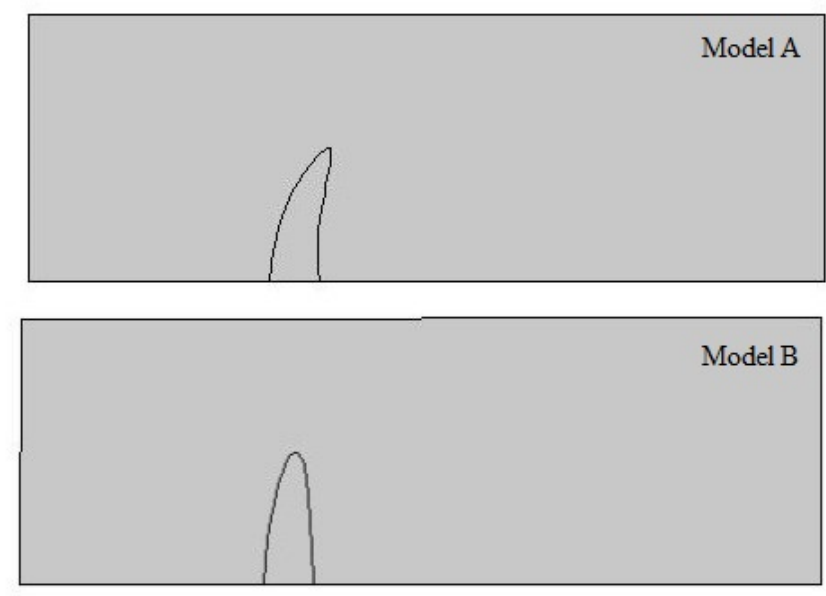

FIGURE V. COMPUTING DOMAIN'S COMPARISON OF MODEL A AND MODEL B

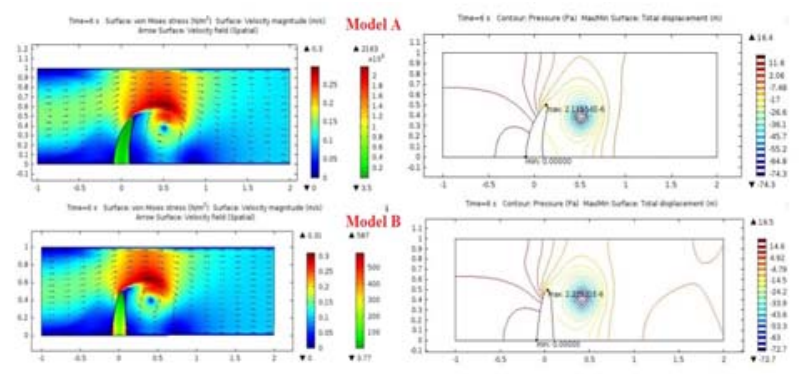

FIGURE VI. COMPARISON OF TWO MODELS' VORTEX STREET AND PRESSURE WHEN T IS $6 S$

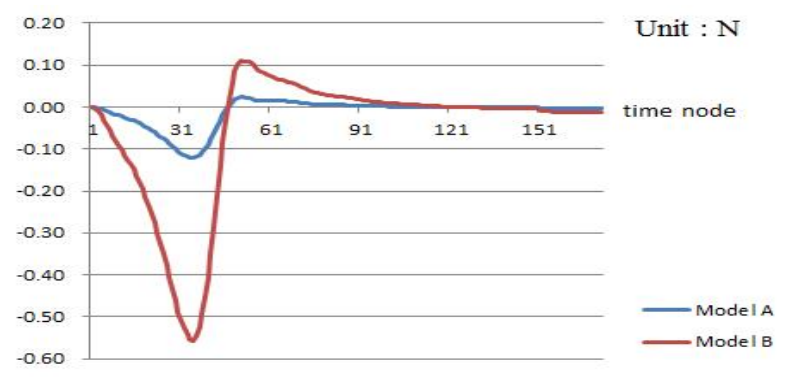

FIGURE VII. COMPARISON OF RESISTANCE SUFFERED BY TWO KINDS OF PECTORAL

\section{CONCLUSION}

This study focused on the Prionace glauca pectoral fins and examined vortex characteristics of pectoral fins in the fluid-solid coupling calculation. Calculation was carried out by the method of transient analysis and dynamic mesh, with a result of obtaining more accurate vortex variation characteristics of pectoral fins in the fluid. Calculations show that:

1. The Vortex flow produced by pectoral fins is more and more large with the increase of the flow rate, and the adaptive contraction modification of the pectoral fins improved the stability of Prionace glauca in the fluid.
2. Special appearance of Prionace glauca pectoral fins helps reduce the flow resistance, slow the impact suffered by pectoral fins, and improve the stability of Prionace glauca during swimming.

This paper verified that the pectoral fins play an important role in keeping balance and stability of Prionace glauca during swimming. The further study requires in-depth study of the flow field's calculation in the three-dimensional model, forming more accurate simulation results.

\section{ACKNOWLEDGEMENT}

This research was financially supported by the National Science Foundation of China(51309150) and Shanghai Municipal Science and Technology Innovation Project (15DZ1202504).

\section{REFERENCES}

[1] McPhail S., Autosub6000: A deep diving long range AUV, Journal of Bionic Engineering [J], 6(1):55-62, 2009, 6(1):55-62.

[2] Jun B.H., Park J.Y., Lee F.Y., Lee P. M. , Lee C. M., Kim K., Lim Y.K., Oh J.H., Development of the AUV 'ISiMI' and a Free Running Test in an Ocean Engineering Basin [J], Ocean Engineering,36(1):2-14, 2009,36(1):2-14.

[3] Nakamura, I., Watanabe, Y., Papastamatiou, Y. P., Sato, K., Meyer, C.G., Yo-yo Vertical Movements Suggest a Foraging Strategy for Tiger Sharks Galeocerdo Cuvier [J]. Mar. Ecol. Prog. Ser. 424:237-246. 2011

[4] Bhalla A P S, Bale R, Griffith B E, et al. A unified mathematical framework and an adaptive numerical method for fluid-structure interaction with rigid, deforming, and elastic bodies [J]. Journal of Computational Physics, 2013, 250(10): 446-476.

[5] Pengtao Sun, Jinchao Xu, Lixiang Zhang. Full eulerian finite element method of a phase field for fluid-structure interaction problem [J]. Computers \& Fluids, 2014, 90(2): 1-8.

[6] Cai Y R, Bi S S, Low K H, et al. Posture analysis and application of a bionic pectoral foil[C]//IEEE International Conference on Robotics and Biomimetics. Piscataway, NJ, US: IEEE, 2011:1783-1788.

[7] Zhou C L, Low K H. Better endurance and load capacity: An improved design of manta ray robot (RoMan-II)[J]. Journal of Bionic Engineering, 2010, 7(S1): S137-S144.

[8] Lang A W, Motta P, Hidalgo P, et al. Bristled shark skin: A micro geometry for boundary layer control. Bioinspi-ration \& Biomimetics, 2008, 3: 46005 